\title{
Determining the mode of operation of the system section, taking into account the current topology and predictive analysis of the active-adaptive network of 6-10 kV
}

\author{
Alexander Yurov ${ }^{1, *}$, Alexander Voronov $^{2}$, and Alexey Lukonin ${ }^{3}$ \\ ${ }^{1}$ Don State Technical University, Don Engineering Center, 344000, Rostov-on-Don, Russia \\ 2 «AC EnergyCom» LLC, 344000 Rostov-on-Don, Russia \\ ${ }^{3}$ «Rosseti» PJSC, Rostov enterprise of main electric networks, 344000, Rostov-on-Don, Russia
}

\begin{abstract}
Technical solutions aimed at improving the reliability of power supply to consumers of the $6-10 \mathrm{kV}$ network are proposed, a block diagram of a fundamentally new device for diagnosing and protecting cells of 6-10 $\mathrm{kV}$ power cable lines with a single algorithm of operation is shown, which allows simultaneously performing current protection of the controlled feeder and monitoring with a predictive assessment (residual resource) of the state of the cable insulation material. A possible scheme of interaction of elements of an active-adaptive power unit of $6-10 \mathrm{kV}$ is shown on the example of a cell of a $6-10 \mathrm{kV}$ cable line. he device will allow you to control leakage currents in distribution networks, taking into account the remaining cable life, reduce insulation losses during power transmission and increase the energy efficiency of the controlled network by reducing power interruptions to the consumer. The quantitative values of the device will be determined after its introduction into trial operation.
\end{abstract}

\section{Introduction}

Currently, the development of the Russian electric power system is determined by a number of areas, including within the framework of the Concept of PJSC "Rosseti" "Digital Transformation 2030". This concept provides for a number of programs to modernize individual areas of the electrical network and create a distributed automation system. The distributed automation system should provide for the individually-centrolyzed principle of building the control of the primary equipment of the power center [1]. At the same time, it is envisaged to create dispatching points (DP) as an automation object, operational control and maintenance through the existing operational dispatch groups (ODG). EDC and DP should exist within the same unified information space [2]. The development of the network should be carried out taking into account the requirement of scaling, that is, with an increase in the size and volume of the network, integration into the hardware and software part of the monitoring, protection, automation, predictive control and management complex $[3,5]$. Development must be carried out with minimal time, money and hardware connections.

*Corresponding author: yurov_dstu@ @ rambler.ru 
Separately, it should be noted the problem of aging and failure of insulation of power cable lines of 6-10 kV distribution networks [4].

\section{Methods and materials}

Insulation failure is one of the most common causes of accidents and loss of consumer power, which is not beneficial (from an economic point of view) to all participants in the electricity market. Power cable line (CL) is the most unreliable element of the distribution network and requires additional control and analysis. The scheme of interaction of network elements is shown in fig. 1.

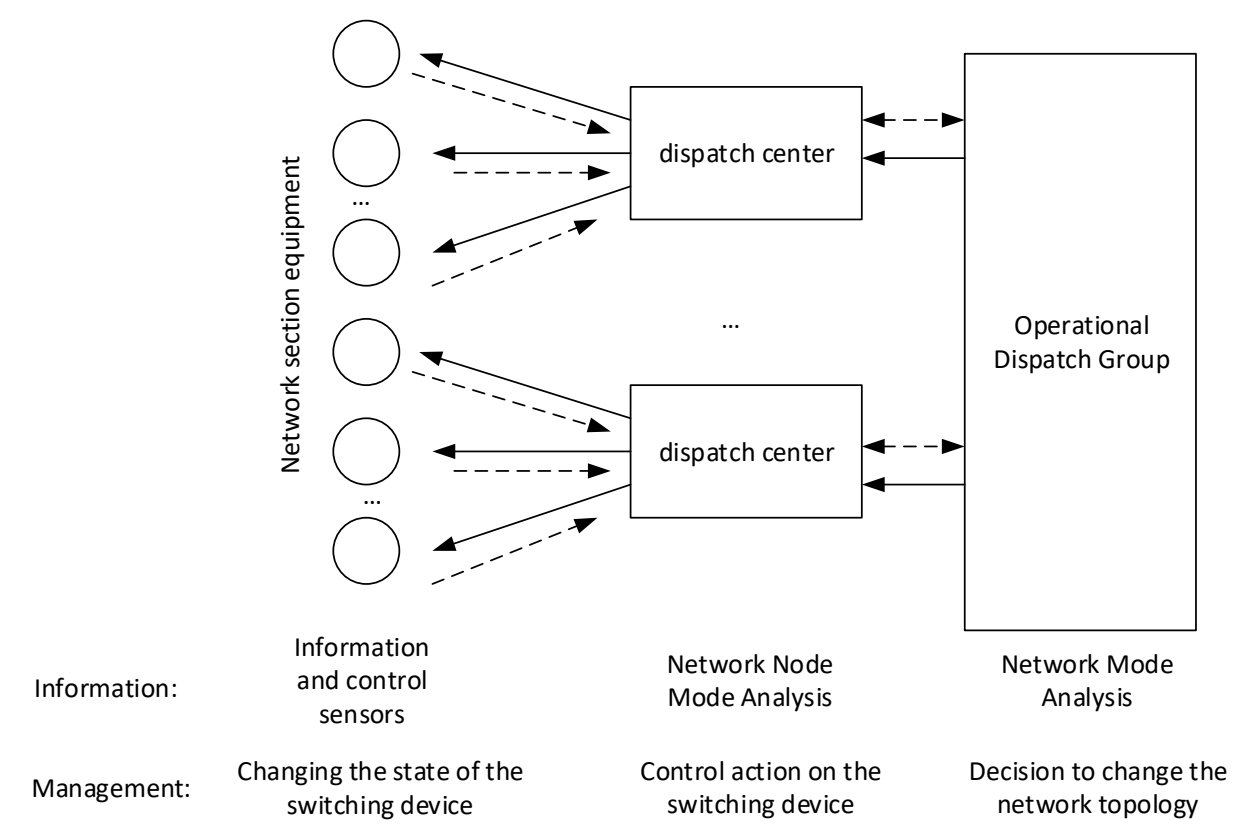

Fig. 1. Schematic of the functional connections of the elements of the electrical network

The primary equipment of the power system must support operation with digital primary information sensors (current transformers and voltage transformers, sensors for monitoring and controlling the condition of equipment), including short-circuit sensors and provide information transfer to the control room with conversion of output protocols in accordance with the IEC 61850 standard [6,7]. In addition, this equipment should be equipped with protection against short-circuit currents, a predictive control system and an uninterruptible power supply, which will increase the reliability of power supply and the functioning of the network, by increasing the observability of operational parameters and controllability of the power unit. An approximate diagram of the interaction of the elements of a node of an activeadaptive network of 6-10 kV is shown in Fig. 2. In order to reduce the number of devices in a cell, their functional connections and mutual influence on each other, it is necessary to develop a microprocessor device (MPU) for complex on-line monitoring of the state of a 6$10 \mathrm{kV}$ cell under operating voltage and converting output protocols in accordance with the IEC61850 standard. The device under development must connect and control data from digital information sensors, namely (current and voltage sensors, equipment monitoring and control sensors). It is necessary to propose an algorithm for the operation of the MPU, taking into account its multifunctionality, a large amount of controlled data, possible malfunctions and methods of monitoring the state of an intelligent electronic device (IED) [8,9]. In the 
device under development, the following basic functions must be implemented for the cell of the cable line 6-10 kV:

- function of the residual life of the cable line. Control of sensors for monitoring and control of the state of insulation and sheath of the power cable line, assessment of the residual life of the operating condition of the cable line in order to adjust the repair schedule (predictive analysis). It is assumed that the monitoring and control system for the signal will work (it is possible to perform the shutdown algorithm with current control, as in software).

- protection functions,

- automation functions,

- instantaneous value measurement functions,

- counters and recorders.

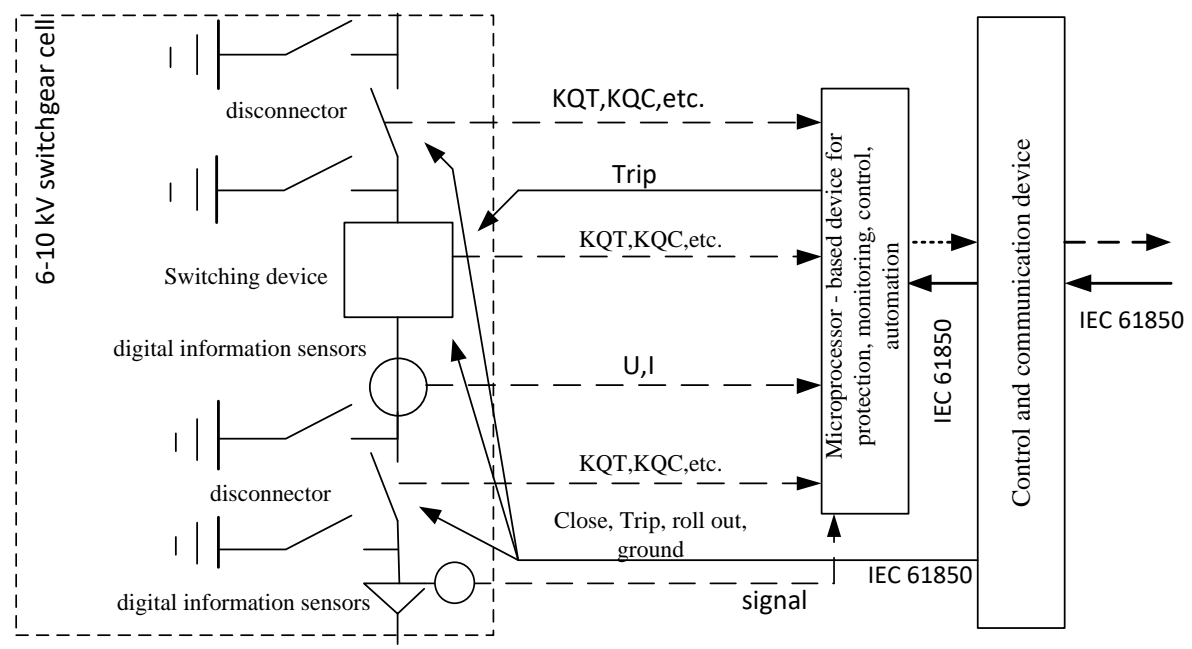

Fig.2. Scheme of interaction of elements of an active-adaptive network node

Equipment and IEDs of power centers and dispatching centers of power centers should automatically calculate the network efficiency mode in real time, classify it according to the main parameters (normal mode, overload, asymmetric load, ground fault, etc.). Make an instant decision on the possibility of the existence of such a regime according to the criteria of trouble-free operation of the power section, monitor the wear and residual life of the equipment referred to this item. It should be noted that not today there is a lack of scientific and technical solutions for the hardware of domestic manufacturers that allow solving the tasks of predictive analysis, protection and automation of equipment with one intelligent electronic device.

The equipment of the operational dispatching group should perform a number of new tasks for the power system: restore power supply to consumers without the participation of operating personnel in the event of an unscheduled shutdown of the power center; monitor the state of the equipment of the power center; Formation of applications for the withdrawal of equipment repairs; direction of the operational-departure brigade; preparation of documentation for admission and work of personnel; maintaining the schedule of equipment input / output, etc.

\section{Results and discussion}

Such a structure of the power system has existed for a long time, the modern direction of development of the power system presupposes a high share of responsibility to assign to 
digital decision-making and system monitoring systems [10,11]. Reducing cross-links and functional impacts will simplify the setup of such devices, reduce the likelihood of personnel errors and increase the circuit and hardware reliability of the substation and the power supply of the network node, reduce the number of emergency shutdowns and wear of the main switching equipment, which will certainly affect energy efficiency and energy saving (reducing depreciation costs).

One of the important directions in the development of the decision-making system is the improvement of the existing and the development of new methods for assessing and identifying emergency and abnormal modes. For this, it is necessary to create adaptive models of a section of the electric power system, taking into account the current topology of the distribution network and the parameters of the elements. The use of computational models will make it possible to clearly predict the new mode and quickly rebuild the network topology in the event of an emergency mode. This direction must meet the requirements of speed, reliability and simplicity of the analysis of the functioning algorithm for control by the operator at the initial stage of implementation in trial operation.

\section{Conclusion}

With the development of digital technologies in the electric power industry, the use of intelligent electronic devices (IED) and remote methods of collecting transmitting and controlling information, the observability (control) of the distribution network increases, its automation and the ability to adapt to operating conditions, which in turn increases the reliability of power supply to consumers of electric energy. Minimization of human resources in the assessment of the residual resource of the distribution network, reduces the labor costs of teams of electrical laboratories, eliminates the human factor of erroneous conclusion of the state of the network, additionally allows you to adjust the schedule for the output of electrical equipment for repair, thereby reducing possible power interruptions to consumers or the number of switches in the distribution network. These factors indicate the need to develop such intelligent combined systems (devices) for protection and monitoring of 6-10 $\mathrm{kV}$ network connections. The device allows you to control the leakage currents of insulation in distribution networks in the range from $1 \mathrm{~mA}$ to $100 \mathrm{~A}$, taking into account the remaining cable life with a connection length of up to $10 \mathrm{~km}$, reduce insulation losses during power transmission and increase the energy efficiency of the controlled network by reducing power interruptions to the consumer. The measured range practically makes it possible to additionally monitor single-phase earth faults, which are frequent for networks with isolated non-mains and cause certain difficulties in detecting them. The quantitative values of the device will be determined after its introduction into trial operation, by calculating the economic profit of electricity market participants by reducing the undersupply of electricity.

\section{References}

1. Mathebula, V.C., Saha, A.K., Multi-state IEC-61850 Substation Communication Network based on Markov partitions and symbolic dynamics, Sustainable Energy, Grids and Networks 26,100466, (2021)

2. Chen, P., Xiao, Z., Yan, P., Wang, X., Wang, L, Journal of Physics: Conference Series, 1871(1), 012041, (2021)

3. Chen, L., Li, H., Charton, T., Zhang, R. Energies 14(8), 2337, (2021)

4. Zhang, J., Guo, Y., Xu, M., Shen, Y., Zhang, K., IOP Conference Series: Earth and Environmental Science, 692(2), 022032, (2021) 
5. Liu, Q., Liu, H., Li, J., Research on digital design and control technology of secondary circuit in substation, IEEE 4th Conference on Energy Internet and Energy System Integration: Connecting the Grids Towards a Low-Carbon High-Efficiency Energy System, EI2 2020, 9347030, 569-574 (2020)

6. Yan, Y., Li, S., Zhang, J., Ye, G., Wang, Z. Research on the Application of Internet of Things in Operation and Management of Distribution Sub-stations, 2020 5th International Conference on Power and Renewable Energy, ICPRE 2020, 9233127, c. 356-360

7. Pinchukov, P., Makasheva, S., Estimation of Digital Substation Reliability Indices, Advances in Intelligent Systems and Computing, 1115 AISC, 3-14 (2020)

8. Mirgaleeva, A.R., Gilfanov, K.H., E3S Web of Conferences, 124,05038 (2019)

9. Development of digital substation-challenges and expectations in utility context, Water and Energy International, 62(5), 19-20 (2019)

10. Kumar, S., Das, N., Islam, S., High voltage substation automation and protection system based on IEC 61850, Australasian Universities Power Engineering Conference, AUPEC, 8757995 (2018)

11. Ferreira, R.D.F., De Oliveira, R.S., Cloud IEC 61850 A Case Study of a Software Defined Protection, Automation Control System, IEEE International Conference on Emerging Technologies and Factory Automation, ETFA, 2018-September, 8502512, 75-82 (2018) 